\title{
Optical in situ calibration of Sb to grow disordered GaInP by MOVPE
}

\author{
Enrique Barrigón ${ }^{\mathrm{a}, *}$, Laura Barrutia ${ }^{\mathrm{a}}$, Ignacio Rey-Stolle ${ }^{\mathrm{a}}$ \\ ${ }^{a}$ Instituto de Energía Solar, Universidad Politécnica de Madrid, Avda. Complutense 3028040 Madrid, \\ Spain
}

\begin{abstract}
We employed Reflectance Anisotropy Spectroscopy (RAS) to obtain the specific molar flow of Sb needed to grow GaInP with a given order parameter by MOVPE. The RAS signature of GaInP surfaces exposed to different $\mathrm{Sb} / \mathrm{P}$ molar flow ratios were recorded. It was determined that the RAS peak at $3.02 \mathrm{eV}$ showed a feature sensitive to the amount of Sb on the surface. The range of $\mathrm{Sb} / \mathrm{P}$ ratios in which $\mathrm{Sb}$ acts as a surfactant was determined using the $\mathrm{RA}$ intensity at $3.02 \mathrm{eV}$. A batch of different GaInP layers were grown using different $\mathrm{Sb} / \mathrm{P}$ ratios and the order parameter of the resulting layers was measured by PL at $20 \mathrm{~K}$. This procedure might be extensible to the calibration of surfactant mediated growth of other materials with characteristic RAS signatures.
\end{abstract}

Keywords:

GaInP, Sb, Surfactant, Reflectance Anisotropy Spectroscopy

\section{Introduction}

GaInP, a key material for many optoelectronic devices such as solar cells, shows CuPttype ordering on the group III-sublattice [1]. In essence, CuPt-type ordering describes a superlattice of alternating Ga- and In-rich $\{111\}$ planes (i.e., $\mathrm{Ga}_{0.5(1-\eta)} \operatorname{In}_{0.5(1+\eta)} \mathrm{P} / \mathrm{Ga}_{0.5(1+\eta)} \operatorname{In}_{0.5(1-\eta)} \mathrm{P}$ ), where $\eta$ is defined as the order parameter. For a fully disordered GaInP material $\eta=0$, whereas for a completely ordered material $\eta=1$. So far, the range for the order parameter for MOVPE-grown GaInP extends from $\eta=0$ to $\eta=0.6$, i.e. no perfectly ordered alloy has been obtained $[2,3]$.

The degree of order modulates, among others, the energy bandgap $\left(\mathrm{E}_{g}\right)$ of the alloy [4]. In this respect, a decrease in the order paramenter (low $\eta$ ) leads to a higher $\mathrm{E}_{g}$. Thus, it is customary to control the ordering of the alloy when it is employed in any optoelectronic device. In order to modulate $\eta$, either typical epitaxial growth parameters (such as growth temperature, growth rate, V/III ratio, doping level, wafer offcut) are changed $[5,6,7,8,9]$ or surfactants are employed during epitaxial growth [10].

\footnotetext{
*Corresponding author

Email address: enrique.barrigon@ies-def.upm.es (Enrique Barrigón)
} 
Surfactants are elements with a low solubility in the solid together with a low vapor pressure that tend to accumulate at the surface during the epitaxial growth [11]. In this way, they affect the growth kinetics and, in turn, the properties of the layer grown. It has been reported that surfactants can reduce the free energy of the epilayer [12], change the surface reconstruction [13] and affect the step kinetics [14]. In MOVPE growth, three different elements have been so far identified that are surfactants on GaInP, namely Te, Bi and $\mathrm{Sb}$. However, $\mathrm{Sb}$ is the most versatile candidate since Te is a n-type dopant on GaInP with a strong memory effect [15], and Bi deteriorates the surface morphology and might form compounds as BiAs and $\mathrm{BiP}[10]$. Sb acts by reducing the amount of $\mathrm{P}$ dimers on the surface, which is the driving force for CuPt ordering [16]. In addition, Sb is isoelectronic with $\mathrm{P}$ (i.e., it will not affect the Fermi level position) and the incorporation of $\mathrm{Sb}$ in the solid is low, namely around $1 \times 10^{18} \mathrm{~cm}^{-3}$ [16]. As an important drawback, if excesive Sb is used in the growth, the resulting GaInP layer will exhibit composition modulation [10] or triple period order [17] which, in turn, lowers notably the value of $\mathrm{E}_{g}$.

The first step to control the degree of order of $\mathrm{GaInP}$ with $\mathrm{Sb}$ is thus to precisely determine the $\mathrm{Sb}$ molar flow that has to be introduced during the growth of GaInP in the reactor chamber to achieve a desired order parameter. Since it is intended that $\mathrm{Sb}$ acts as a surfactant and that it does not significantly incorporate into the semiconductor lattice, the molar flow should be rather low. Additionally, it is important to notice that the adequate molar flow lies within a specific range to get the desired surfactant effect (i.e., to get a disordered GaInP layer). Below this range no effect is expected, whereas above, the previously commented triple period ordering is induced [17]. Throughout the text, the $\mathrm{Sb}$ molar flow is going to be expressed in terms of the $\mathrm{Sb} / \mathrm{P}$ ratio in the gas phase, in parts per million (ppm). If we take a look to the values published in the literature, there is a broad range of values. For instance, Ref. [16] reports a $\mathrm{Sb} / \mathrm{P}$ ratio of $100 \mathrm{ppm}$ to disorder the GaInP whereas in Refs. [18],[19] and [20] the values are 40, 300 and $800 \mathrm{ppm}$, respectively. The differences between the reported values probably rely on the process T, V/III ratio and MOVPE reactor geometry.

Instead of performing time-consuming, trial-and-error iterations to determine the optimal $\mathrm{Sb} / \mathrm{P}$ ratio to tune the order parameter for a specific reactor and set of growth conditions, we employed Reflectance Anisotropy Spectroscopy (RAS) [21] to this end. In brief, RAS is a highly surface-sensitive spectroscopic tool which has been applied to the study of semiconductor surfaces $[22,23]$, domain quantification [24], desorption studies [25] and even to monitor reactive ion etching [26]. So far, RAS [27, 28] and surface photoabsorption (SPA) [29] spectroscopic measurements have been applied to the study of the $\mathrm{CuPt}_{B}$-type ordering of GaInP, establishing a clear correlation between surface reconstruction during growth and the resulting degree of order, depending on V/III ratio [7, 28], growth $\mathrm{T}[27,6,28]$ and substrate offcut [5]. However, only SPA has been used to the in situ monitoring of Sb adand desorption on GaInP $[30,16]$. In this paper, we use RAS to calibrate the molar flow range of $\mathrm{Sb}$, that will be later employed to grow GaInP layers with different $\eta$ values. 


\section{Experimental}

Samples were grown in a horizontal commercial MOVPE reactor (Aixtron AIX200/4) equipped with an in situ RA spectrometer (LayTec EpiRAS 200). We used Ge(100) substrates with $6^{\circ}$ misorientation towards [011] direction and the Sb precursor employed was TESb. A growth T of $675^{\circ} \mathrm{C}$, a V/III ratio of 120 and a growth rate of $0.60 \mathrm{~nm} / \mathrm{s}$ were used to grow these layers. More details on the GaInP growth can be found in Ref. [31].

\section{Results and discussion}

A calibration GaInP sample with a thickness of $1.1 \mu \mathrm{m}$ was firstly grown. In this way, we have a GaInP virtual substrate where the optical in situ calibration of Sb can take place. Right after the GaInP growth, we measured a RA spectrum as a benchmark (see black RA spectrum in Fig. 1) under a constant molar flow of $\mathrm{PH}_{3}\left(1.79 \times 10^{-2} \mathrm{~mol} / \mathrm{min}\right)$ and at $675^{\circ} \mathrm{C}$ (growth T). Subsequently, different molar flows of Sb were introduced in the reactor chamber. For each molar flow, the RAS signal was recorded twice to detect possible changes in the surface. Nonetheless, both RA spectra were virtually identical for each $\mathrm{Sb} / \mathrm{P}$ case (except for the highest $\mathrm{Sb} / \mathrm{P}$ ratio introduced), which indicates that the adsorption of $\mathrm{Sb}$ at the surface takes place within some seconds, as stated in Ref. [32]. Fig. 1 summarizes all the spectra recorded for a set of different $\mathrm{Sb} / \mathrm{P}$ ratios introduced, as shown in the legend. Please note that all the RA spectra were taken from a non-growing surface at the same temperature, namely $675^{\circ} \mathrm{C}$. As the RAS setup only records signals in absolute value due to the wafer rotating system in our MOVPE reactor, the sign of the RA spectra has been corrected by comparison to the spectra published in Refs. [27, 28].

Generally speaking, all the RA spectra in Fig. 1 show two main features, namely one asymmetric, negative peak at $2.00 \mathrm{eV}$ and a positive peak at $3.02 \mathrm{eV}$ with a tail that expands towards higher photon energies. The black line in Fig. 1 corresponds to the benchmark RA spectrum with no Sb. The red RA spectrum corresponds to the case where the amount of $\mathrm{Sb}$ introduced in the chamber (a $\mathrm{Sb} / \mathrm{P}$ ratio of $56 \mathrm{ppm}$ ) produces no significant change in the RA spectrum. On the contrary, as long as we increase the $\mathrm{Sb} / \mathrm{P}$ ratio between 411 and $1720 \mathrm{ppm}$, the feature at $3.02 \mathrm{eV}$ starts to decrease whereas the negative peak at $2.00 \mathrm{eV}$ remains unaltered. This tendency continues until a $\mathrm{Sb} / \mathrm{P}$ ratio of $3150 \mathrm{ppm}$ is introduced (pink curve), where the feature at $3.02 \mathrm{eV}$ does not change but the feature at $2.00 \mathrm{eV}$ decreases and the tail at higher energies increases.

One of the main properties of the elements acting as surfactants is that they can be easily re-evaporated from the surface. Accordingly, the RAS signal should be recovered once the $\mathrm{Sb}$ is removed from the reactor chamber. To illustrate this point, Fig. 2(a) shows the normalized RAS signal in transient mode at $3.02 \mathrm{eV}$ - the energy at which the biggest differences were observed - of the same GaInP surface as in Fig. 1, when the Sb is added to and removed from the reactor (as indicated by the dotted vertical lines). The RAS signal is normalized so that the surface free of Sb has a signal near unity. Fig. 2(a) shows that $\mathrm{Sb}$ induces a reversible process at the surface since, i.e., once the $\mathrm{Sb}$ is switched off, the previous level of the RAS signal is recovered. At $t=270 \mathrm{~s}, \mathrm{Sb}$ was added to the reactor at a 
$\mathrm{Sb} / \mathrm{P}$ ratio of $1720 \mathrm{ppm}$. At $\mathrm{t}=370 \mathrm{~s}$ the $\mathrm{Sb}$ was removed and the signal gradually recovers the value before $\mathrm{Sb}$ introduction. In order to study the reproducibility of the process, after some minutes the same amount of $\mathrm{Sb}$ is introduced in the chamber. The signal goes again to the same value previously observed.

On the contrary, if an amount of $\mathrm{Sb} / \mathrm{P}$ as high as $3150 \mathrm{ppm}$ is added to the reactor for enough time, not only the RA spectrum is slightly different (see pink curve in Fig. 1) but also the surface is irreversibly changed. Fig. 2(b) illustrates this case when a $\mathrm{Sb} / \mathrm{P}$ ratio of $3150 \mathrm{ppm}$ is introduced. The signal monotonically increases, indicating that the surface is continuously changing. At $\mathrm{t}=1350 \mathrm{~s}$, the $\mathrm{Sb}$ is removed from the reactor. In this case, the surface does not recover its reconstruction previous to Sb introduction, but the signal goes to zero, indicating that an irreversible process took place at the surface.

In other words, the experimental observations of Fig. 1 and 2 indicate that the Sb introduced behaves as a surfactant since 1) it induces changes in the surface which can be monitored with RAS (i.e., reduction of the RAS feature at $3.02 \mathrm{eV}$ with $\mathrm{Sb}$ molar flow) and 2) shows a reversible behavior. As reported in Ref.[28], the RAS feature at $3.02 \mathrm{eV}$ is useful to establish a correlation between the during-growth surface dimer reconstruction and the resulting degree of order. Besides, this feature in SPA measurements is related to the presence of [110] P-dimers on the surface [29]. The observations made by the two characterization techniques are essentially coincident, since after $\mathrm{P}$ desorption the $(2 \mathrm{x} 1)$ surface reconstruction turns into a $(2 \times 4)$ configuration $[28,33]$.

Accordingly, by monitoring the changes at $3.02 \mathrm{eV}$ of the RAS signal we are able to determine the adequate $\mathrm{Sb}$ molar flow range that potentially will modulate the order parameter of $\mathrm{GaInP}$ grown at $675^{\circ} \mathrm{C}$ without degrading the surface, i.e., a Sb/P ratio from 411 to $1720 \mathrm{ppm}$. In order to proof this point, several Zn-doped GaInP layers were grown with different molar flows of $\mathrm{Sb}$ within such range. The specific $\mathrm{Sb} / \mathrm{P}$ used are the ones determined in Fig. 1, excluding the $\mathrm{Sb} / \mathrm{P}$ ratio of $3150 \mathrm{ppm}$ which induced irreversible effects at the surface. Although Sb enhances Zn incorporation [34], the layers show a doping value in the range of 1 to $4 \times 10^{17} \mathrm{~cm}^{-3}$ which does not significantly affect $\eta[3]$.

Fig. 3 shows the $\eta$ parameter obtained for the GaInP layers with different $\mathrm{Sb} / \mathrm{P}$ ratios in the gas phase, as indicated in the legend of the figure. The estimation of $\eta$ comes from the dependence of the energy bandgap of an alloy with its order parameter [35]:

$$
E_{g}(\eta)=E_{g}(\eta=0)-\eta^{2} \Delta E_{g}
$$

where $\Delta E_{g}$ is the bandgap reduction of the fully ordered alloy, which has been experimentally determined to be $471 \mathrm{meV}$ [2]. Accordingly, the order parameter can be inferred from photoluminescence (PL) measurements:

$$
\eta=\sqrt{\frac{1991-\mathrm{PL} \text { at } 20 \mathrm{~K}(\mathrm{meV})}{471}}
$$

where $1991 \mathrm{meV}$ is the estimated energy bandgap of a completely disordered GaInP at $20 \mathrm{~K}$ grown lattice-matched on a Ge substrate. This value is corrected from the value reported for GaInP on GaAs, assuming that the decrease in the bandgap of the GaInP when growing 
on a Ge substrate is the same as that at RT, namely, $14 \mathrm{meV}$. The PL measurements were thus performed at $20 \mathrm{~K}$, excited with a $532 \mathrm{~nm}$ laser of $26 \mathrm{~mW}$. As can be seen in Fig. 3, the order parameter is reduced as the amount of $\mathrm{Sb}$ is increased - within the $\mathrm{Sb} / \mathrm{P}$ molar flow range as determined Fig. 1-, confirming the surfactant effect of Sb [16]. The $\eta$ value of the sample grown with no Sb turns out to be 0.53, which indicates that the GaInP is highly ordered for the given growth conditions. Once $\mathrm{Sb}$ is used during the GaInP growth, the lowest value of $\eta$ achieved is 0.30. Similar results were reported in Ref. [19] for Sb-mediated growth of GaInP on singular and vicinal GaAs substrates, which are also plotted in Fig. 3 for direct comparison.

Finally, Fig. 3 also evidences the high values of $\mathrm{Sb} / \mathrm{P}$ that were used in comparison to other values in the literature. Such differences are mainly due to the differences in the temperature used in the process and in reactor configuration, since Sb readily evaporates from the surface at the temperature employed in our study. Indeed, the growth temperature used for this experiment is $675^{\circ} \mathrm{C}$, which is $55^{\circ} \mathrm{C}$ higher than the temperatures used in Ref. [19].

\section{Summary and conclusions}

In summary, RAS stands out as a valuable tool to calibrate the Sb molar flow needed to modulate the order parameter of GaInP grown by MOVPE. For our reactor geometry and at $675^{\circ} \mathrm{C}$, RAS signal at $3.02 \mathrm{eV}$ was employed to identify an useful $\mathrm{Sb} / \mathrm{P}$ range between 411 and $1720 \mathrm{ppm}$, whereas for higher $\mathrm{Sb} / \mathrm{P}$ values the surface is irreversibly modified. An effective change in the order parameter of $\mathrm{GaInP}$ layers - grown with $\mathrm{Sb} / \mathrm{P}$ values within the range detected by RAS - has been obtained.

\section{Acknowledgement}

Financial support from the EC (NGCPV-283798), the spanish MINECO (TEC201237286, TEC2011-28639-C02-01 and IPT-2011-1408-420000) and CAM(S2099/ENE1477) is gratefully acknowledged. The technical assistance of Jesús Bautista is also acknowledged. 


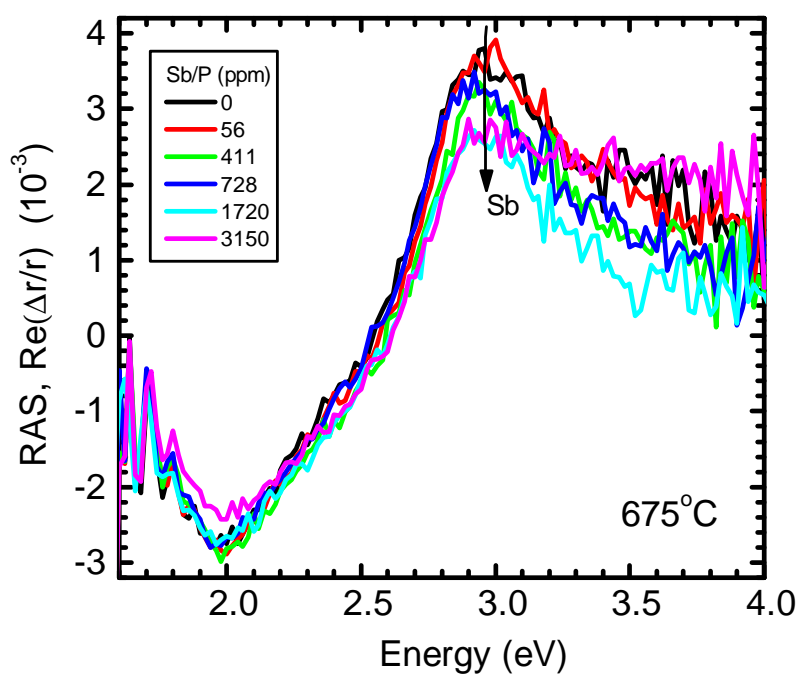

Figure 1: In situ RA spectra of a GaInP surface exposed to different $\mathrm{Sb} / \mathrm{P}$ ratios at $675^{\circ} \mathrm{C}$.
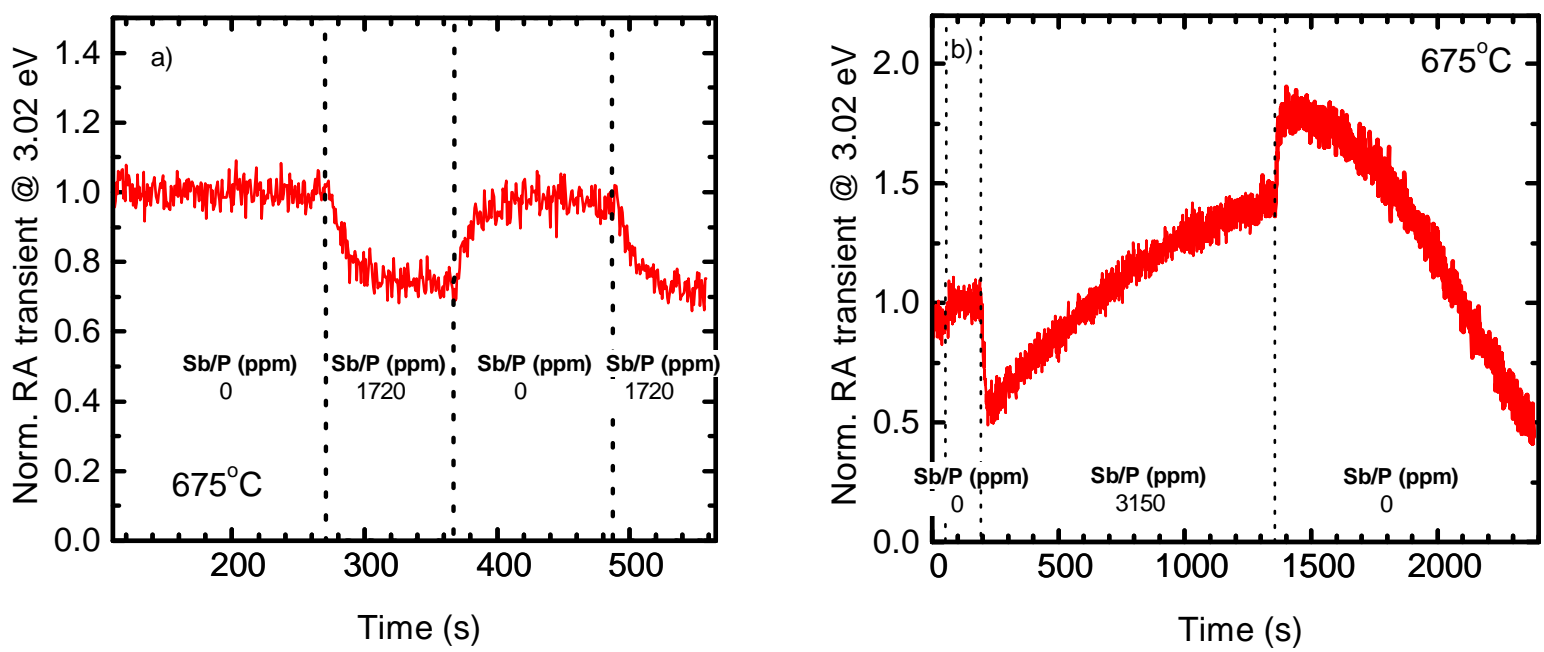

Figure 2: Normalized RA transient at $3.02 \mathrm{eV}$ of a GaInP surface as TESb is added to and removed from the reactor, when the amount of $\mathrm{Sb}$ introduced produces reversible (a) and irreversible (b) changes on the surface 


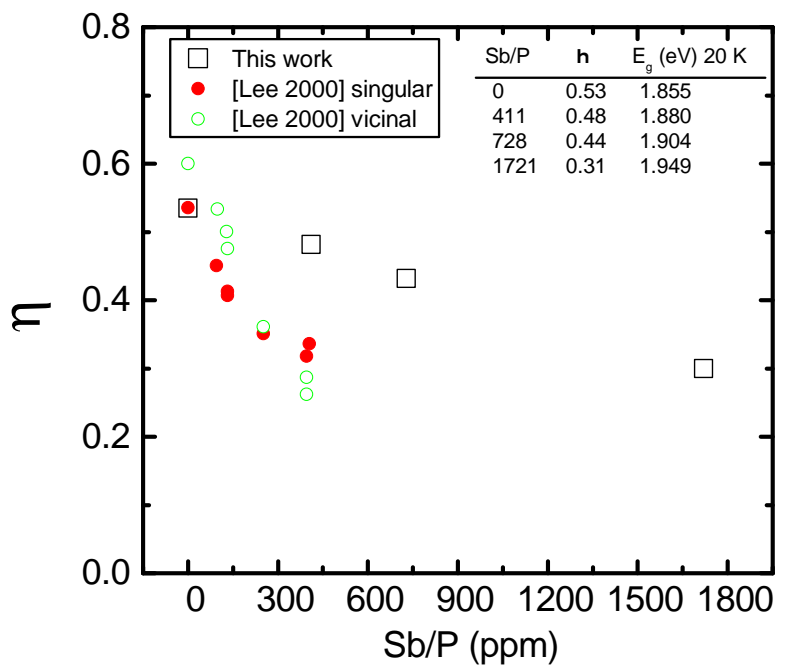

Figure 3: Order parameter versus the $\mathrm{Sb} / \mathrm{P}$ ratio in the vapor phase for the samples grown. The data from [19] is included as a reference. The inset shows the value of the PL peak energy @ $20 \mathrm{~K}$.

\section{References}

[1] A. Gomyo, T. Suzuki, K. Kobayashi, S. Kawata, I. Hino, T. Yuasa, Appl. Phys. Lett. 50 (1987) 673.

[2] P. Ernst, C. Geng, Appl. Phys. Lett. 67 (1995) 2347.

[3] S. H. Lee, C. M. Fetzer, J. Appl. Phys. 86 (1999) 1982.

[4] A. Gomyo, T. Suzuki, S. Iijima, Phys. Rev. Lett. 60 (1988) 2645-2648.

[5] H. Murata, I. H. Ho, Y. Hosokawa, G. B. Stringfellow, Appl. Phys. Lett. 68 (1996) 2237-2239.

[6] H. Murata, I. H. Ho, L. C. Su, Y. Hosokawa, G. B. Stringfellow, J. Appl. Phys. 79 (1996) 6895.

[7] H. Murata, T. Hsu, I. Ho, L. Su, Y. Hosokawa, G. Stringfellow, Appl. Phys. Lett. 68 (1996) 1796.

[8] S. R. Kurtz, J. M. Olson, A. Kibbler, Appl. Phys. Lett. 57 (1990) 1922.

[9] I. García, I. Rey-Stolle, C. Algora, W. Stolz, K. Volz, J. Cryst. Growth 310 (2008) $5209-5213$.

[10] G. Stringfellow, J. Shurtleff, R. Lee, C. Fetzer, S. Jun, J. Cryst. Growth 221 (2000) $1-11$.

[11] M. Copel, M. C. Reuter, E. Kaxiras, R. M. Tromp, Phys. Rev. Lett. 63 (1989) 632-635.

[12] N. Grandjean, J. Massies, V. H. Etgens, Phys. Rev. Lett. 69 (1992) 796-799.

[13] F. K. LeGoues, V. P. Kesan, S. S. Iyer, J. Tersoff, R. Tromp, Phys. Rev. Lett. 64 (1990) 2038-2041.

[14] S. Lee, C. Fetzer, J. Appl. Phys. 85 (1999) 3590.

[15] S. Lee, C. Fetzer, G. Stringfellow, J. Cryst. Growth 195 (1998) $13-20$.

[16] J. K. Shurtleff, R. T. Lee, C. M. Fetzer, G. B. Stringfellow, Appl. Phys. Lett. 75 (1999) 1914-1916.

[17] C. M. Fetzer, R. T. Lee, J. K. Shurtleff, G. B. Stringfellow, S. M. Lee, T. Y. Seong, Appl. Phys. Lett. 76 (2000) 1440-1442.

[18] J. Olson, W. McMahon, S. Kurtz, 4th IEEE World Conference on Photovoltaic Energy Conversion 1 (2006) 787-790.

[19] R. Lee, J. Shurtleff, C. Fetzer, G. Stringfellow, S. Lee, T. Seong, J. Appl. Phys. 87 (2000) 3730-3735.

[20] T. Y. Seong, S. M. Lee, R. T. Lee, G. B. Stringfellow, Surf. Sci. 457 (2000) L381 - L385.

[21] P. Weightman, D. S. Martin, R. J. Cole, T. Farrell, Rep. Prog. Phys. 68 (2005) 1251-1341.

[22] J.-T. Zettler, Prog. Cryst. Growth Charact. Mater. 35 (1997) $27-98$.

[23] U. Rossow, L. Mantese, D. Aspnes, Appl. Surf. Sci. 123 (1998) 237-242.

[24] H. Döscher, T. Hannappel, B. Kunert, A. Beyer, K. Volz, W. Stolz, Appl. Phys. Lett. 93 (2008).

[25] E. Barrigón, S. Brückner, O. Supplie, P.Kleinschmidt, I. Rey-Stolle, T. Hannappel, Appl. Phys. Lett. 102 (2013) 111608-111608-4. 
[26] L. Barzen, J. Richter, H. Fouckhardt, M. Wahl, M. Kopnarski, Applied Surface Science 328 (2015) 120 -124 .

[27] J. S. Luo, J. M. Olson, S. R. Kurtz, D. J. Arent, K. A. Bertness, M. E. Raikh, E. V. Tsiper, Phys. Rev. B 51 (1995) 7603-7612.

[28] M. Zorn, P. Kurpas, A. I. Shkrebtii, B. Junno, A. Bhattacharya, K. Knorr, M. Weyers, L. Samuelson, J. T. Zettler, W. Richter, Phys. Rev. B 60, 11 (1999).

[29] H. Murata, I. Ho, Appl. Phys. Lett. 67 (1995) 3747.

[30] J. Shurtleff, R. Lee, C. Fetzer, G. Stringfellow, S. Lee, T. Seong, J. Cryst. Growth 234 (2002) 327 336.

[31] E. Barrigón, B. Galiana, I. Rey-Stolle, J. Cryst. Growth 315 (2011) 22-27.

[32] J. Shurtleff, R. Lee, G. Stringfellow, IEEE International Symposium on Compound Semiconductors (2000) $197-203$.

[33] P. Vogt, K. Lüdge, M. Zorn, M. Pristovsek, W. Braun, W. Richter, N. Esser, Phys. Rev. B 62 (2000) $12601-12604$.

[34] D. Chapman, A. Howard, G. Stringfellow, J. Cryst. Growth 287 (2006) $647-651$.

[35] W. Su-Huai, D. B. Laks, A. Zunger, Appl. Phys. Lett. 62 (1993) 1937. 